\title{
La dimension territoriale comme condition à la « nouvelle ruralité »
}

\author{
Jean Ruegg \\ Université de Lausanne
}

\section{Introduction}

Notre propos est fort proche, dans le fond, du projet initié par les organisateurs du séminaire «Multifonctionnalité des activités, pluralité des identités » qui s'est tenu en décembre 2003 à Paris ${ }^{1}$. En effet, la multifonctionnalité est un des concepts clefs de la nouvelle ruralité. Il apparaît dans la foulée des réformes que plusieurs politiques agricoles nationales connaissent dès la fin des années 1980. De même, le territoire comprend une composante identitaire forte, du moins si nous le comprenons comme une entité spatialement délimitée offrant un lieu de participation, de débat et de décision pour les acteurs qui l'ont produit, qui se le sont approprié et qui s'emploient à l'entretenir.

Les lignes qui suivent constituent alors une sorte de nouvelle variation à partir d'une interrogation que nous avons commencée à développer et à formaliser dans deux publications récentes $^{2}$. L'interrogation résulte de notre perspective qui est celle de l'aménagiste avant tout, soucieux de l'utilisation précautionneuse de la ressource rare qu'est le sol. Nous sommes conscients du rôle indéniable et largement sous-estimé que les paysans ont joué en Suisse pour freiner l'étalement urbain. Mais, comme cette fonction est une conséquence - et non pas un objectif explicite - de l'ancienne politique agricole mise en place au sortir de la Seconde guerre mondiale, nous nous demandons quels seront les effets territoriaux des comportements induits par la nouvelle politique agricole suisse de la fin des années 1990. Plus précisément, nous doutons que le milieu des aménagistes puisse encore longtemps compter sur le soutien des agriculteurs pour limiter l'urbanisation.

Le premier texte reprenait, notamment, certains travaux de Raffestin ${ }^{3}$. Nous défendions l'idée que tout effort pour limiter l'accroissement des surfaces bâties suppose l'existence d'acteurs capables de lui opposer des projets de préservation du sol qui soient viables économiquement et acceptables socialement. Nous prônions alors la redécouverte de stratégies intégratives mêlant mieux les préoccupations urbaines et environnementales, à l'image du programme de la CPRE (Campaign to Protect Rural England). Dans le second, et en reprenant cette fois le concept d'agriculture urbaine proposé par Fleury et Donadieu ${ }^{4}$, nous situions l'enjeu central de la problématique dans l'identification et la définition d'un lieu de négociation et de médiation pour que l'agriculture devienne un outil de l'aménagement de la ville. Nous nommions ce lieu le " projet ville-campagne». Ici, nous aimerions poursuivre dans la même veine. La multifonctionnalité et la nouvelle ruralité sont évidemment des notions intéressantes et qui mériteraient à elles seules un examen approfondi et une analyse attentive. Pour notre part, elles ne font sens que si elles permettent de redécouvrir des modalités pour « faire territoire ». Au risque de la caricature, nous prétendons en effet volontiers que l'évolution récente de la politique agricole a justement délaissé la dimension territoriale pour assumer prioritairement une tâche sociale. Il s'agit de construire un modèle permettant aux agriculteurs restant en activité de tirer de leur exploitation un revenu décent. Et tant pis si cela se traduit par une baisse massive du nombre des exploitants et des exploitations, voire une réduction significative de la surface agricole utile. Sans le souci du territoire, un tel dessein pourrait être une fuite en avant plutôt qu'une proposition durable.

Le présent article comprend trois volets. Nous montrerons d'abord comment la politique agricole suisse induit des limites au principe de la multifonctionnalité. La multifonctionnalité constitue un enjeu impor- 
tant pour caractériser les relations nouvelles qui se développent entre ville et campagne, entre territoires bâtis et non bâtis, entre paysages urbanisés et paysages ouverts propices, par exemple, à la détente et aux loisirs des populations urbaines. Mais, seule, elle ne peut suffire. Nous pensons qu'il est indispensable de lui associer une dimension territoriale, surtout s'il s'agit de tenir compte de l'exigence du développement durable et de l'utilisation mesurée de ressources comme l'énergie ou le sol. Nous porterons ensuite notre attention sur des pratiques ou des sensibilités originales susceptibles de recréer du lien social - et donc de faire du territoire - non pas à l'échelle du monde ou du globe, mais à celle des régions. Elles ont pour nom agriculture contractuelle de proximité ou souveraineté alimentaire, par exemple, et visent à remettre au centre du débat les questions liées à la détermination du juste prix des produits et à l'identification des destinataires des services fournis par l'agriculture. Elles relèvent de stratégies où il s'agit à la fois d'aller vers des produits qui incorporent plus de valeur et vers des mécanismes sociaux mieux à même de faire reconnâ̂tre l'existence de ces valeurs complémentaires. Dit autrement, il s'agit donc de stratégies locales ou régionales susceptibles d'intéresser le bénéficiaire à payer un prix plus équitable. Ces stratégies sont essentielles pour contrer, en dernier ressort, ce que Berque nomme la «forclusion $»^{5}$. La forclusion rend compte de notre tendance à omettre le travail - et donc à ignorer le juste prix - qui est derrière les paysages que, par ailleurs, nous valorisons tant. La forclusion a cela de pernicieux, et donc de dangereux, qu'elle nous incite à considérer le paysage comme un acquis pérenne, comme un élément de décor qui nous serait donné et dans lequel nous pourrions nous mouvoir indéfiniment. Pourtant il est bien plus sage et prudent de considérer le paysage comme un territoire fruit d'un travail ou produit de relations sociales. Pour donner une tournure plus concrète à nos propos, nous terminerons en indiquant comment le canton de Genève s'engage dans cette voie et promeut ce type d'approche en recourant à la fois à de nouvelles lois, à la promotion de label comme "Genève région Terre avenir» et au soutien d'initiatives comme l'« Affaire Tournerêve ».

\section{Politique agricole suisse, multifonctionnalité et déterritorialisation}

La politique agricole suisse contemporaine est élaborée au lendemain de la Seconde guerre mondiale.

Pour œuvrer au maintien d'une population paysanne qui soit la plus forte possible, elle est associée à la Défense nationale. L'agriculture joue un rôle majeur en termes d'approvisionnement du pays. Elle doit notamment permettre de subvenir aux besoins des habitants en cas de fermeture des frontières. Mener une telle politique réclame toutefois une modification de la Constitution fédérale. Elle intervient en 1947. Le nouvel article constitutionnel permet à la Confédération d'intervenir dans l'économie agricole. Le dispositif prévoit que les prix des produits agricoles doivent couvrir les frais de production et assurer un revenu décent aux paysans. Ce dernier doit être un « revenu paritaire "), où la notion de parité découle d'un parallèle que la politique agricole introduit avec la rémunération journalière de l'ouvrier. Sur cette base, l'aide financière de la Confédération est ciblée vers le soutien des prix à la production, la garantie de l'écoulement des produits et l'octroi de subventions à l'exportation de certains biens (fromage essentiellement). Ces différents éléments sont précisés dans la Loi fédérale sur l'agriculture (LAgr) de 1951.

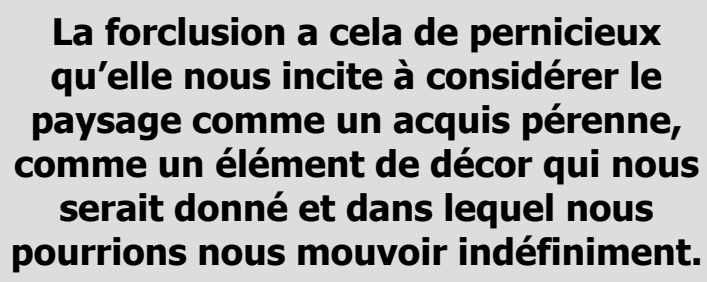

Par rapport à la problématique qui nous intéresse ici, ces préliminaires sont importants pour mettre en exergue une fonction essentielle, même si elle est indirecte, que la politique agricole va exercer en termes d'aménagement du territoire. Elle soustrait une part importante de la superficie du pays de la pression à l'urbanisation En effet, le mécanisme de soutien des prix à la production vaut uniquement pour des modes de production qui sont «tributaires du sol». Cette expression, helvético-suisse sans doute, désigne exclusivement des modes de production qui incorporent la terre en tant que matière première. Sont donc écartés du dispositif les cultures hors-sol ou les élevages de type industriel, par exemple. Mais si la quantité des biens agricoles produits selon des modes de production tributaires du sol influence directement l'aide de la Confédération et donc la hauteur du revenu paysan, l'intérêt évident des milieux agricoles est donc d'obtenir la protection des terres agricoles. Ce vœu est exaucé avec la Loi fédérale sur l'aménagement du ter- 
ritoire (LAT). Dès son entrée en vigueur en 1980, la LAT oblige toutes les autorités compétentes (municipalités ou cantons) à désigner comme zone agricole (art. 16 LAT) une part conséquente du territoire de leur juridiction. Et, pour parfaire l'édifice, il est entendu que les terrains alors placés en zone agricole ne sont pas des réserves pour l'urbanisation future. La zone agricole rassemble les surfaces requises pour mener à bien les objectifs de la politique agricole et pour assurer la pérennité économique des exploitations agricoles ${ }^{6}$.

Cependant, dès le début des années 1990, l'échafaudage patiemment et méthodiquement construit vacille. Pour des raisons à la fois interne et externe au pays (coût de l'intervention étatique pour le citoyen-contribuable et accroissement de la pression internationale en faveur de la libéralisation des échanges), la Confédération va devoir reprendre l'ensemble de sa politique agricole. Cette réorientation se traduit par un amendement de la Constitution, la définition d'une nouvelle loi sur l'agriculture (1998) et la révision de la loi sur l'aménagement du territoire (1999). Il en résulte une modification profonde des composantes du revenu paysan. Désormais le revenu agricole dépend de trois sources distinctes. La première reste liée au produit dégagé par la vente de la production. Mais le prix n'est plus soutenu par la Confédération. Il doit tendre vers le prix du marché de référence (qui est, en l'occurrence, celui de la politique agricole commune (PAC) élaborée par l'Union européenne). La seconde résulte de paiements directs. Il s'agit de subventions versées par la Confédération en contrepartie de prestations d'intérêt général - souvent non ou partiellement marchandes - fournies par l'agriculteur. La troisième, qui entre en ligne de compte si les deux premières ne suffisent pas, rend possible la réalisation sur l'exploitation d'activités accessoires susceptibles de générer un revenu complémentaire (infrastructure pour l'agritourisme, atelier de réparation de machines agricoles, bureau d'informatique pour le développement de programmes de comptabilité agricole, par exemple). La nouvelle politique agricole introduit donc des changements substantiels quant à la définition même de l'activité agricole. Le premier, qui est aussi le plus évident, provient du passage d'une agriculture monofonctionnelle à une agriculture multifonctionnelle. Autrefois rattachée au seul secteur primaire, l'agriculture intègre désormais des activités des secteurs secondaires (transformation de denrées alimentaires sur l'exploitation, agriculture semi indus- trielle, culture hors-sol) et tertiaire (vente directe). Le second est moins souvent évoqué. Son implication pour l'aménagement du territoire est toutefois considérable. L'activité agricole ne se définit plus par des modes de production qui sont tributaires du sol. Il suffit que la nature agricole du produit soit établie pour que la conformité à l'article 16 LAT soit reconnue.

\section{Désormais le revenu agricole dépend de trois sources distinctes.}

En termes d'utilisation du sol et de structure agricole, nous assistons alors à plusieurs phénomènes. Si la surface agricole utile varie encore assez peu (diminution de l'ordre de $3 \%$, entre 1984 et 1997), le nombre d'agriculteurs et d'exploitations diminue fortement (63 600 exploitations en 2005 contre 92800 en 1990, selon les chiffres de l'Office fédéral de la statistique). Quant à la taille moyenne de ces dernières, elle augmente régulièrement pour s'établir à 16.7 ha en moyenne, en 2005. En outre, pour améliorer l'efficacité et la rentabilité de leur domaine, les paysans sont parfois amenés à construire de nouvelles infrastructures (hangars, écuries, halles d'engraissement, serres, silos) ou à réaliser des aménagements propices à l'acquisition de revenus accessoires (construction ou transformation de ruraux en logement ${ }^{7}$, en structure d'accueil pour l'agritourisme ou en écuries pour des chevaux en pension, par exemple). Contrairement au régime qui prévalait sous l'ancienne loi agricole, ils deviennent donc des acteurs qui participent à l'urbanisation des zones agricoles. Quantitativement parlant, cette nouvelle tendance est encore peu visible. Elle n'est pas suffisante pour représenter déjà un sujet de débat et un enjeu politique. Mais elle ne peut pas non plus être négligée pour deux raisons au moins. Il est attendu que la situation des paysans continuera à se détériorer. L'enjeu pour la Confédération est en effet de parvenir à poursuivre son soutien à l'agriculture avec des fonds restreints et une forte diminution de la protection douanière. Dans ce contexte, elle a déjà annoncé dans un texte publié en 2005 ("Politique agricole $2011 »)$ qu'elle est prête à ce que le nombre des agriculteurs et des exploitations continue à régresser. Nul ne peut exclure à terme que là où la pression sur la zone agricole est la plus forte, des agriculteurs renoncent à leur activité - et donc au rôle qu'ils jouent pour contrer l'urbanisation - pour profiter des plusvalues foncières qu'un changement d'affectation du 
sol générerait. Mais, surtout, la fragilité de l'ensemble du système semble provenir de l'écart croissant qui caractérise les relations entre les agriculteurs et les non-agriculteurs. Avec la chute des effectifs agricoles, la population suisse qui habite à plus de $75 \%$ dans des agglomérations urbaines perd sa fibre et ses racines paysannes. Elle peine toujours plus à comprendre les revendications du monde agricole. Contrairement à la période des Trente glorieuses, elle est en tout cas moins encline à les partager ${ }^{8}$. Cette situation est d'autant plus sérieuse que le lien social se délite, d'une part, et que la politique agricole est menée presque uniquement au niveau de la Confédération, d'autre part. Ainsi, les produits agricoles sont le plus souvent orientés vers un marché structuré à l'échelon national par des filières relativement longues dont les paysans n'ont d'ailleurs pas la maîtrise. Le consommateur les retrouve transformés via le réseau des deux principaux distributeurs du pays (Coop et Migros).

\section{La souveraineté alimentaire se différencie de la sécurité alimentaire.}

Quant à la multifonctionnalité ou à la rémunération des valeurs non marchandes procurées par l'agriculture - comme le maintien de paysages ouverts à proximité des lieux d'habitation - ce sont des thèmes qui sont régulièrement accaparés par l'Office fédéral de l'agriculture. Il les utilise pour justifier la politique agricole en général et les paiements directs en particulier que la Confédération concède aux paysans pour leur venir en aide. Les liens ne peuvent donc que se distendre et la dimension territoriale s'estomper. $\mathrm{Au}$ mieux, ils sont entretenus d'une manière indirecte par le biais justement de la part de l'impôt sur les personnes physiques qui est dédiée au financement de la politique agricole de la Confédération ou par le biais des votations populaires qui portent sur des objets relevant de cette politique. Pourtant, nous sommes convaincus que le maintien durable des activités susceptibles de freiner l'étalement urbain passe par la réappropriation par les agriculteurs et les non-agriculteurs des relations qui les rassemblent. L'une des voies propices à cette réappropriation est d'inviter les bénéficiaires des externalités positives fournies par l'agriculture à s'interroger sur leur juste prix et à exprimer leur demande et leurs préférences en rémunérant directement ceux qui peuvent les leur fournir. De tels projets existent. Avec la vente directe de produits agricoles dans des circuits courts et la fourniture de services dans le domaine du tourisme rural, l'agriculture contractuelle de proximité, fondée sur le modèle français de l'Association pour le maintien de l'agriculture paysanne (AMAP), en fournit un bon exemple. Malheureusement, elle reste trop souvent confidentielle. Elle procède d'initiatives réalisées par quelques « convaincus ». D'ailleurs le secteur agricole luimême tend à la considérer comme secondaire, voire marginale. Cette réappropriation des relations entre agriculteurs et non-agriculteurs mérite alors des stratégies mieux structurées. D'abord, elle doit pouvoir être spatialement ancrée en offrant aux principaux intéressés une certaine proximité géographique. Et puis, elle mérite d'être articulée à d'autres préoccupations qui, pour simplifier, sont celles du développement durable et de son fameux slogan " think globally, act locally ». Enfin, elle a tout à gagner à s'inscrire dans un cadre institutionnel que seule la présence de l'acteur public rend possible. À ces conditions, qui dépassent la seule notion de multifonctionnalité, cette réappropriation a des chances d'incorporer la dimension territoriale et de «faire territoire ». Telle est la perspective dans laquelle nous proposons de présenter le concept de la souveraineté alimentaire.

\section{Nouvelles approches : la souveraineté alimentaire}

La souveraineté alimentaire se différencie de la sécurité alimentaire. Si toutes deux traitent de la meilleure manière de subvenir aux besoins alimentaires d'un pays ou d'une région, elles divergent quant aux conceptions qui les fondent. "[La sécurité alimentaire domine]. Elle postule qu'un pays n'a pas besoin d'une agriculture autosuffisante. Ce qu'il doit faire, c'est produire du pouvoir d'achat là où il est le plus compétent pour se procurer la nourriture dont il a besoin là où elle se trouve. [...]. La souveraineté alimentaire consiste à promouvoir l'autosuffisance alimentaire et à s'en remettre aux importations seulement en deuxième recours $»{ }^{9}$.

Élément intéressant, la souveraineté alimentaire est proposée par le syndicat international Via Campesina à l'occasion du Sommet mondial de l'alimentation en 1996. Elle rassemble des adhérents issus aussi bien des pays du Sud que du Nord et se démarque très clairement des approches néo-libérales et des thèses dominantes prônées par l'Organisation mondiale du commerce (OMC). 
Pour d'aucuns, la souveraineté alimentaire traduit une visée réactionnaire et protectionniste puisqu'elle favorise l'agriculture de proximité au détriment des échanges internationaux et des fameux préceptes issus de la théorie des avantages comparatifs. À bien des égards, pourtant, elle rejoint de nombreuses préoccupations altermondialistes formulées depuis longtemps par des spécialistes du développement et de l'histoire économique. Sous l'égide cette fois de la préoccupation environnementale et du développement durable, la Revue Durable lui consacre deux dossiers en 2003 et 2006. Outre les questions du développement et du commerce équitable, une agriculture fondée sur la souveraineté alimentaire est également propice à la réduction des flux de matière et à l'atténuation de la pression que les activités humaines exercent sur la planète.

Il ne s'agit pas ici d'en faire l'apologie. Elle nous intéresse plutôt dans la mesure où, par rapport à ce besoin de territorialisation évoqué ci-dessus, la souveraineté alimentaire invite à la fois à revenir sur la question du juste prix des produits et des aménités fournies par l'agriculture et à proposer que les paysans et les consommateurs négocient ensemble, à une échelle territoriale locale ou régionale, ce que pourrait être ce " fameux juste prix ». Elle a donc le potentiel de remettre en question la situation qui prévaut en Suisse. À l'heure actuelle, en effet, ces questions ne sont pas abordées directement par les acteurs concernés. Mais elles sont «intermédiées », via les paiements directs, par la politique agricole de la Confédération, laquelle est bien davantage alignée sur la stratégie de la sécurité alimentaire. La souveraineté alimentaire est donc une piste possible pour une réappropriation par le consommateur de la traçabilité de son alimentation, de la connaissance des filières d'approvisionnement et, par extension, de la production et du maintien du territoire dans lequel il habite. Tandis que, du côté du producteur, elle est un moyen envisageable pour refaire du lien et transmettre ses préoccupations (ferme pédagogique, vente directe), de faire reconnaître son travail et, le cas échéant, d'être pleinement intégré et associé aux débats qui influenceront les orientations agricoles futures ${ }^{10}$.

La souveraineté alimentaire, par la démarche qu'elle implique, fournit donc une bonne illustration de ce nouveau partenariat territorialisé qu'il s'agit de promouvoir entre consommateur-urbain et agriculteur. Mais là ne réside pas son seul intérêt. Pour clore, nous aimerions montrer que la souveraineté alimentaire ne suppose pas forcément une action militante. À Genève, en effet, elle est reprise dans la stratégie que le canton développe dans le cadre de la mise en œuvre du développement durable.

\section{La souveraineté alimentaire est donc une piste possible pour une réappropriation par le consommateur de la traçabilité de son alimentation.}

\section{Exemple genevois}

Le territoire du canton de Genève est l'un des plus exigus des cantons suisses $\left(282 \mathrm{~km}^{2}\right)$. Il abrite en revanche une population relativement importante, de l'ordre de 400000 habitants. Essentiellement connu en tant que canton urbain, Genève joue pourtant un rôle tout à fait significatif en termes de production agricole. Près de la moitié de son territoire lui est dédiée. Ses 480 exploitants gèrent une véritable ceinture verte, établie comme telle dès les années 1930. La préservation à des fins agricoles d'une surface qui est d'autant plus significative que la pression à l'urbanisation est forte $^{11}$ doit beaucoup aux agriculteurs eux-mêmes et à la Chambre genevoise de l'agriculture. Ensemble, ils ont su freiner l'urbanisation.

Cette situation particulière explique alors peut-être pourquoi le cas genevois continue à être unique en Suisse, avec notamment une présence forte des autorités cantonales et des instances professionnelles et politiques en charge de l'agriculture. Parmi les initiatives les plus remarquables, citons d'abord la loi du 21 octobre 2004 dite Loi sur la promotion de l'agriculture (LGePA). Cette loi complète la Loi fédérale sur l'agriculture. Elle ne remet nullement en question les dispositions clefs prévues par la Confédération comme les paiements directs. Elle tente d'infléchir son champ d'application en intégrant des préoccupations que nous qualifierons de préoccupations ancrées ou territorialisées. Non contente de s'inscrire... « [...] dans les principes du développement durable, [la $L o i$ sur la promotion de l'agriculture] a pour but de promouvoir, dans le canton de Genève, une agriculture productrice, rémunératrice, concurrentielle, respectueuse de l'environnement et répondant aux normes sociales et aux besoins du marché et de la population » [art. 1, al. 1]. 
Plus loin, elle précise encore qu'elle vise, en particulier, à : « $[\ldots]$ c) améliorer les conditions d'existence de la population paysanne, ainsi que les conditions de travail des ouvriers agricoles; d) assurer et soutenir la promotion et l'écoulement des produits agricoles genevois; e) sauvegarder la viabilité des espaces ruraux; $[. .$.$] h) favoriser les liens entre la ville et la$ campagne, dans une perspective de plus grande proximité » [art. 1, al. 2].

Elle soutient également « les mesures visant à favoriser les connaissances et l'éducation de la population en matière d'agriculture genevoise » [art. 9, al. 1], les efforts de commercialisation et d'écoulement des produits agricoles genevois, « notamment en vue de l'obtention de prix équitables » [art. 13, al. 1], de même que «les projets ayant pour but la culture, la fabrication, la transformation ou la commercialisation de produits agricoles et agroalimentaires innovants, ainsi que le développement de prestations de services, contribuant à la création d'une valeur ajoutée à l'agriculture $[\ldots] »[$ art. 14, al. 1].

Enfin, elle stipule que « seuls ont droit aux prestations cantonales prévues dans la présente loi les agriculteurs qui n'utilisent pas d'organismes génétiquement modifiés, ni de produits qui en sont issus » [art. 43, al. 1].

Certes, la notion de souveraineté alimentaire n'y est pas nommément citée. Mais c'est chose faite dans le règlement d'application (RGePA) de ladite loi : «Le canton développe et encourage des mesures favorisant une agriculture reposant sur des principes de qualité, d'équité, de traçabillité et de proximité, en adéquation avec la demande des consommateurs, ainsi qu'avec les principes de la souveraineté alimentaire » [art. 5, al. 1 RGePA].

Par rapport à nos thèses, il est donc tout à fait remarquable de trouver une base légale qui reflète parfaitement nos préoccupations, qui définit le canton comme le territoire adéquat pour un nouveau partenariat entre agriculteur et consommateur et qui formalise les modalités de son application en reprenant les termes mêmes de la souveraineté alimentaire.

Si nul n'est censé ignoré la loi, rien n'en garantit pour autant une mise en œuvre automatique et immédiate. Là encore, le canton de Genève est le lieu d'initiatives qui démontrent que le pas est bien franchi. Nous nous limiterons alors à quelques commentaires finaux ayant traits au label " Genève région - Terre avenir » et à l'« Affaire Tournerêve ».

\section{Le canton de Genève est le lieu d'initiatives qui démontrent que le pas est bien franchi.}

En matière de label, il est fréquent que les États interviennent en tant qu'organes de reconnaissance et de régulation. À Genève, l'autorité exerce une fonction supplémentaire puisqu'elle est propriétaire du label «Genève Région - Terre Avenir ». Le label fait l'objet d'un règlement général, administré par le Service cantonal de l'agriculture. Le règlement, dans son premier paragraphe, mentionne dûment le principe de la souveraineté alimentaire de Genève, de même que le rapprochement des paysans et des citadins et le raccourcissement des circuits d'approvisionnement. Le label «Genève Région - Terre Avenir » est donc bien un élément significatif qui concrétise la Loi sur la promotion de l'agriculture (LGePA). Il permet d'associer les milieux agricoles, la grande distribution, les associations de consommateurs et les syndicats agricoles. En 2006, la marque «Genève région - Terre avenir » rassemblait 180 adhérents (y compris Coop Genève et Migros Genève).

Quant à l' "Affaire Tournerêve », il s'agit d'une initiative d'agriculture contractuelle de proximité initiée, à son origine, pour relancer la production d'huile de tournesol sur le territoire genevois. Lancée en 2003, elle a depuis été consolidée avec l'adhésion d'autres agriculteurs qui permettent d'assurer une offre de produits plus diversifiés. Aujourd'hui, cette association alimente plus de 1500 familles genevoises contractantes. Elle complète la coopérative des Jardins de Cocagne qui pratique l'agriculture contractuelle de proximité depuis plus de 25 ans. Comme pour les exemples mentionnés ci-dessus, l' « Affaire Tournerêve » se caractérise par le soutien des acteurs institutionnels (autorités cantonales, service cantonal de l'agriculture, vulgarisation agricole) et des syndicats agricoles (Uniterre, en l'occurrence, qui est aussi membre de Via Campesina $)^{12}$.

\section{Synthèse finale}

Le paysage agricole, en Suisse comme ailleurs, est soumis à de profondes mutations. La multifonctionna- 
lité et la nouvelle ruralité en sont quasiment devenus des termes emblématiques. Nous avons tenté de montrer que, dans le contexte helvétique, la multifonctionnalité a été introduite avant tout pour garantir un revenu décent aux agriculteurs. Cette démarche, pilotée par la Confédération, n'est pas optimale dans la perspective de l'aménagement du territoire. Elle altère la notion même de zone agricole et émousse la capacité de l'agriculture à freiner l'avancée de l'urbanisation. En ce sens, la multifonctionnalité est une notion limitée qui n'est pas entièrement satisfaisante. Afin d'éviter qu'elle ne continue à flotter dans un espace abstrait non directement accessible aux consommateurs et déterminé par la Confédération, les grands distributeurs et les associations agricoles nationales, nous préconisons de l'ancrer, de la territorialiser en lui associant une dimension locale ou régionale.

La notion de souveraineté alimentaire possède un tel potentiel. Elle est d'autant plus intéressante qu'elle est un point de ralliement entre des préoccupations en termes de développement (relations nord-sud) et de protection de l'environnement (réduction des flux de matière). Dans le contexte de la souveraineté alimentaire, la multifonctionnalité permet d'esquisser une «nouvelle ruralité territorialisée ». Le cas genevois permet d'en fournir une illustration probante. Nous y avons montré, certes rapidement, comment les autorités cantonales sont actives à promouvoir de nouvelles lois (Loi sur la promotion de l'agriculture) et de nouvelles initiatives (label « Genève région - Terre avenir », " Affaire Tournerêve ») qui définissent un lieu de médiation pour qu'agriculteurs et consommateurscitadins identifient et conviennent ensemble d'un prix équitable intégrant la rémunération des prestations agricoles non marchandes. C'est bien par ce mécanisme négocié, régulé par l'acteur public, et qui suppose des relations effectives entre partenaires proches, que la présence de la dimension territoriale peut-être assurée. C'est donc bien en cela qu'il est possible de copléter la multifonctionnalité et d'esquisser une nouvelle ruralité... qui soit vraiment nouvelle.

\section{Notes et références}

1 Laurent, C. et J. Rémy (2004). « Multifonctionnalité, activités, identités », Les Cahiers de la multifonctionnalité, $\mathrm{n}^{\circ} 7$, p. 5-15.

2 Ruegg, J. et J. Salomon Cavin (à paraître). « Maîtriser l'étalement urbain : de la stratégie agricole au pas de deux ville-campagne », dans L. Monteventi Weber, Campagne- ville : le pas de deux; enjeux et opportunités des recompositions territoriales, Lausanne, PPUR; Bourdin, D., J. Ruegg et J. Salomon Cavin (à paraître). « De l'agriculture périurbaine au projet ville-campagne : quels enjeux pour la Suisse », texte présenté le 11 octobre 2007 au colloque «Les agricultures périurbaines : un enjeu pour la ville », organisé par l'ENSP et le laboratoire GECKO.

3 Raffestin, C. (1986). «Éléments pour une théorie de la frontière $»$, Diogène, ${ }^{\circ} 134$, p. 3-21

4 Fleury, A. et P. Donadieu (1997). « De l'agriculture périurbaine à l'agriculture urbaine », Le Courrier de l'environnement 31, document de 15 pages disponible en ligne à l'adresse : www.inra.fr/dpenv/fleurc31.htm (site consulté le 8 août 2007).

5 Berque, A. (2005). « La forclusion du travail médial », L'Espace Géographique, vol. 1, n 85, p. 81-90.

6 L'apogée du système est atteinte en 1992 avec le plan sectoriel des surfaces d'assolement. Établi par la Confédération, ce plan fixe pour chaque canton suisse le nombre de mètres carrés de terres agricoles qu'il doit préserver «à tout prix » de l'urbanisation afin de rendre possible les objectifs de la défense nationale en termes d'approvisionnement, principalement.

7 Selon une statistique que l'Office fédéral du développement territorial vient de rendre publique, ce sont, depuis 1990, environ 740 nouveaux logements et bâtiments d'habitation qui sont créés chaque année en zone agricole. Ce chiffre représente $5 \%$ du total des logements réalisés chaque année en Suisse.

8 La première marque de «désamour» date de 1986. À l'instigation des associations de consommateurs, le peuple suisse désavoue pour la première fois ses paysans en rejetant l'arrêté fédéral sur l'économie sucrière indigène.

9 La Revue Durable (2003). «Agriculture : de la nécessité des peuples de se nourrir eux-mêmes », $n^{\circ} 6, p .14$.

10 Nous pensons ici aux questions qui commencent à être discutées en Suisse (et à l'échelon international aussi) et qui concernent la production agricole non alimentaire (agricarburant, exploitation de sous-produits agricoles dans le domaine des cosmétiques, par exemple).

11 L'évolution du territoire genevois ne peut être comprise sans prendre en compte les territoires de la France voisine et du canton de Vaud (district de Nyon). Ces derniers jouent en effet un rôle essentiel pour accueillir la part de la population qui ne trouve pas de logement dans le canton de Genève.

12 Le règlement du label et des informations complémentaires sont disponibles en ligne :

www.terre-avenir.ch ou www.geneve.ch/agriculture (sites consultés le 28 octobre 2007). 


\section{Publicité \\ « 4 carrés »}

